\title{
Doege-Potter Syndrome with a Benign Solitary Fibrous Tumor: A Case Report and Literature Review
}

\author{
Turab Mohammed $^{a} \quad$ Gonca Ozcan $^{a}$ Ayesha S. Siddique ${ }^{b}$ \\ Ronald N. Araneta III ${ }^{\text {b }}$ Dennis E. Slater ${ }^{c}$ Aziz Khan ${ }^{a}$ \\ aDepartment of Internal Medicine, University of Connecticut, Farmington, CT, USA; \\ ${ }^{b}$ Department of Pathology, Hartford Hospital, Hartford, CT, USA; 'Department of \\ Hematology-Oncology, Hartford Hospital, Hartford, CT, USA
}

\section{Keywords}

Doege-Potter syndrome - Non-islet cell tumor hypoglycemia · Insulin-like growth factor II ·

NAB2-STAT6 $\cdot$ Solitary fibrous tumor

\begin{abstract}
Doege-Potter syndrome is a rare paraneoplastic syndrome that is often diagnosed incidentally during the workup of hypoglycemia of unclear etiology. It is characterized by a non-islet cell tumor hypoglycemia secondary to excessive production of partially processed IGF-II hormone from a solitary fibrous tumor (SFT). Often these tumors are intrathoracic, benign, and asymptomatic. Occasionally they present as a paraneoplastic event; hypertrophic osteoarthropathy in Pierre-Marie-Bamberger syndrome and hypoglycemia in Doege-Potter syndrome. The NAB2-STAT6 gene fusion is the hallmark of the SFT. Complete surgical resection of the tumor often results in resolution of symptoms and cure in most cases. Here we present the case of an 83-year-old non-diabetic female with recurrent syncopal events who was diagnosed with the Doege-Potter syndrome secondary to a SFT of pleura. Her tumor was positive for NAB2-STAT6 gene fusion on RT-PCR. Following the resection of the giant tumor mass, she became symptom-free within $24 \mathrm{~h}$, and has remained asymptomatic at 4 months followup.

(C) 2021 The Author(s).

Published by S. Karger AG, Basel
\end{abstract}

\section{Introduction}

Solitary fibrous tumors (SFT) are an uncommon group of neoplasm arising from mesenchymal cells. Doege-Potter syndrome is a rare presenting manifestation of SFTs seen in less than $5 \%$ of patients with SFT. It is a paraneoplastic syndrome with recurrent non-islet cell 


\section{Case Reports in Oncology}
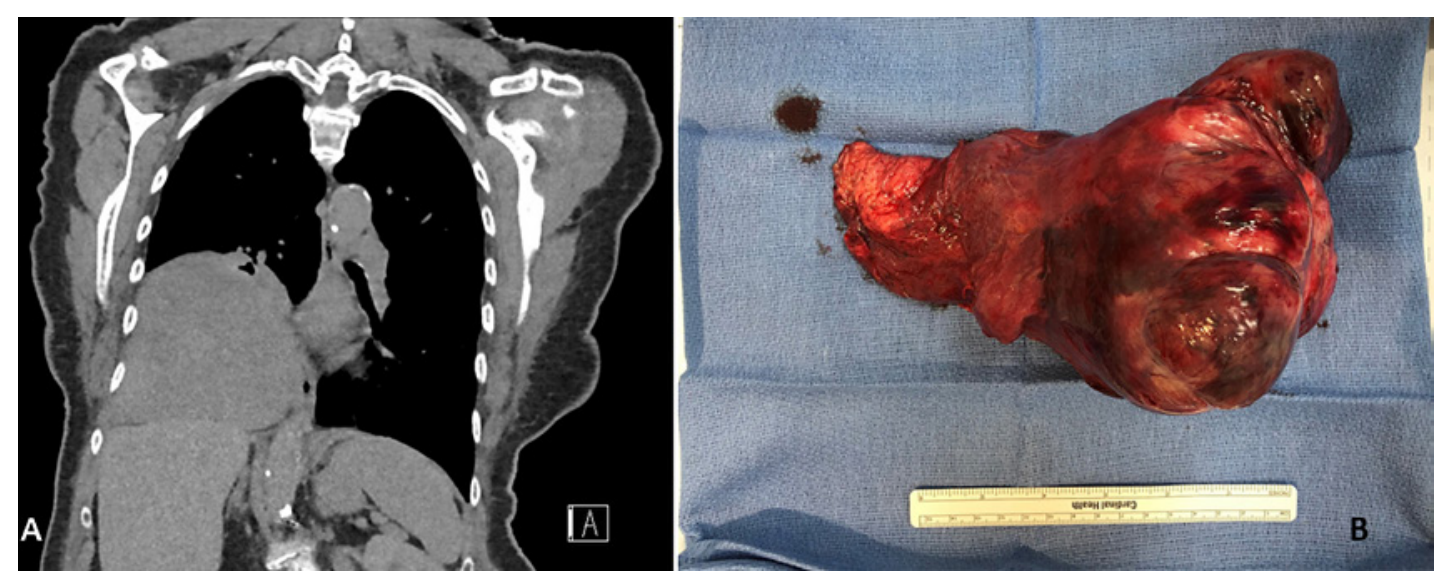

Fig. 1. A Coronal section of the CT chest showing right lower lobe mass abutting adjacent lung, mediastinum and diaphragm below. B Gross appearance of the mass $(>15 \mathrm{~cm})$ post resection.

tumor hypoglycemia (NICTH) owing to excessive secretion of precursor "big" insulin-like growth factor II (IGF-II) from the SFT [1]. This syndrome was first described in 1930 [2, 3], and since then, has been reported only in case reports. To date, no systematic studies exist on this rare clinical entity. SFT of the pleura (SFTP) is a rare subset of pleural tumors that constitute $<5 \%$ of all pleural neoplasms originating from the submesothelial fibroblasts of the visceral pleura. These are often slow-growing, indolent neoplasms (80-85\%) with low malignant potential [4]. Han et al. [5] reported that SFTP was the most common type of SFT associated with Doege-Potter syndrome. NAB2-STAT6 (NGFI-A binding protein 2 [NAB2] gene and signal transducer and activator of transcription 6 [STAT6] gene) fusion results in a chimeric nuclear protein that causes constitutive activation of transcription and abnormal proliferation of cells resulting in tumorigenesis [6].

Here we report a case of Doege-Potter syndrome secondary to SFTP and briefly review the available literature discussing the mechanism of hypoglycemia in patients with such tumors, the diagnosis, and management of Doege-Potter syndrome.

\section{Case Presentation}

An 83-year-old non-diabetic female with a history of hypertension presented with recurrent episodes of lightheadedness and falls for the last month. Her review of systems was negative, and the physical exam did not reveal any significant findings. Her cardiac workup, including a 30-day event monitor and echocardiogram, was unremarkable. Computed tomography (CT) of the head was negative for any intracranial abnormality. Complete blood count was unremarkable, but comprehensive metabolic panel revealed blood glucose of $30 \mathrm{mg} / \mathrm{dL}$ (normal: $>70 \mathrm{mg} / \mathrm{dL}$ ) and serum lipase of $115 \mathrm{U} / \mathrm{L}$ (normal: 13-60 U/L). Biochemical workup for hypoglycemia revealed a low serum free insulin level $0.6 \mathrm{IU} / \mathrm{mL}(1.5-14.9 \mathrm{IU} / \mathrm{mL})$, low proinsulin $<4 \mathrm{pmol} / \mathrm{L}$ ( $<18.8 \mathrm{pmol} / \mathrm{L})$, low serum IGF-1 33 $\mathrm{ng} / \mathrm{mL}$ (34-246 ng/mL), negative screen for blood sulfonylurea and insulin antibodies, a normal ACTH 45 pg/mL (6-50 pg/mL) and an a.m. cortisol level $9.6 \mu \mathrm{g} / \mathrm{dL}(6.2-19.4 \mu \mathrm{g} / \mathrm{dL})$. The IGF-II levels were elevated at $370 \mathrm{ng} / \mathrm{mL}(47-350 \mathrm{ng} / \mathrm{mL})$. A CT chest/abdomen/pelvis was performed, which revealed a large heterogeneous mass within the lower lobe of the right lung (shown in Fig. 1A). Imaging was negative for any adenopathy, other mass lesions,

\section{Karger'}



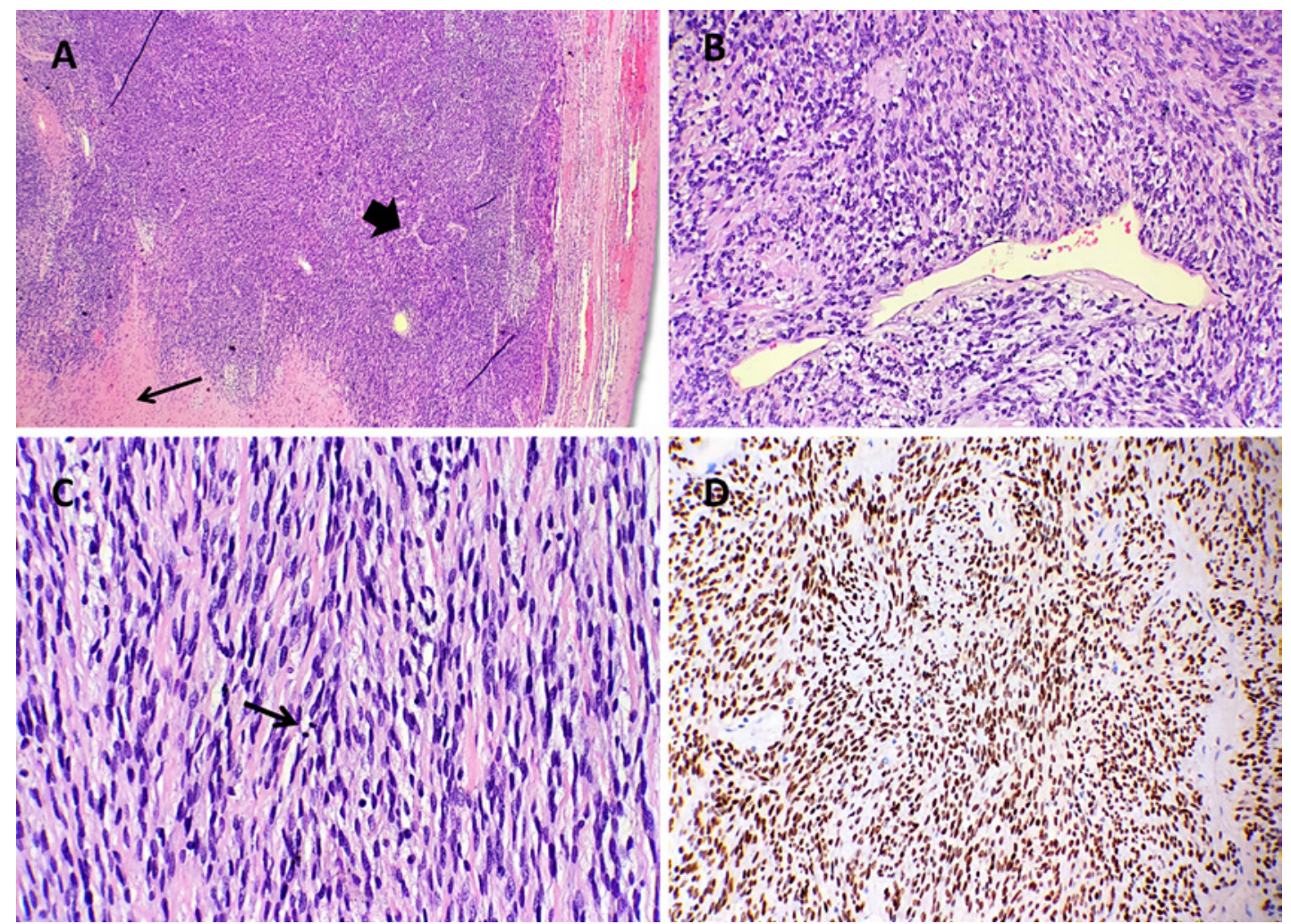

Fig. 2. A Low power showing well-circumscribed, encapsulated proliferation of spindle cells with staghorn vascularity (thick arrow) and areas of hyaline degeneration (thin arrow) but no necrosis. B Staghorn vessels with spindle cells showing mild to moderate nuclear atypia. C Up to 4 mitoses per high-power field. D Strong and diffuse nuclear positivity for STAT6.

or bone metastasis. The bronchoscopic evaluation revealed a friable mass, but the biopsy was inconclusive, and cytology was negative for cancerous cells. Given the high index of suspicion, a CT-guided biopsy was obtained, and it revealed spindle cell neoplasm with mild to moderate atypia without necrosis (shown in Fig. 2A-C). Further immunohistochemistry analysis revealed the cells were positive for STAT6 (signal transducer and activator of transcription 6) and CD34 (shown in Fig. 2D; Fig. 3). Reverse transcriptase-polymerase chain reaction (RT-PCR) confirmed the NAB2-STAT6 fusion. Other pertinent immunostaining including thyroid transcription factor 1 , TTF-1 (for synovial sarcoma), cytokeratin AE1/3, CK-AE1/3 (for carcinoma), desmin, and smooth muscle actin (smooth muscle markers), SRY-Box transcription factor 10, SOX-10 (for melanocytes and schwannocytes lineage) were all negative. The findings of NICTH in the background of SFTP were consistent with the diagnosis of Doege-Potter syndrome. The patient was placed on dextrose $10 \%$ continuous infusion and was instructed to have frequent meals. However, she continued to have recurrent hypoglycemic episodes. She underwent surgical resection of the thoracic mass (shown in Fig. 1B). Her blood sugars improved a few hours post-surgery, and she was taken off the dextrose infusion. Four months post-surgery, the patient has stayed disease-free with no signs of hypoglycemic episodes. 


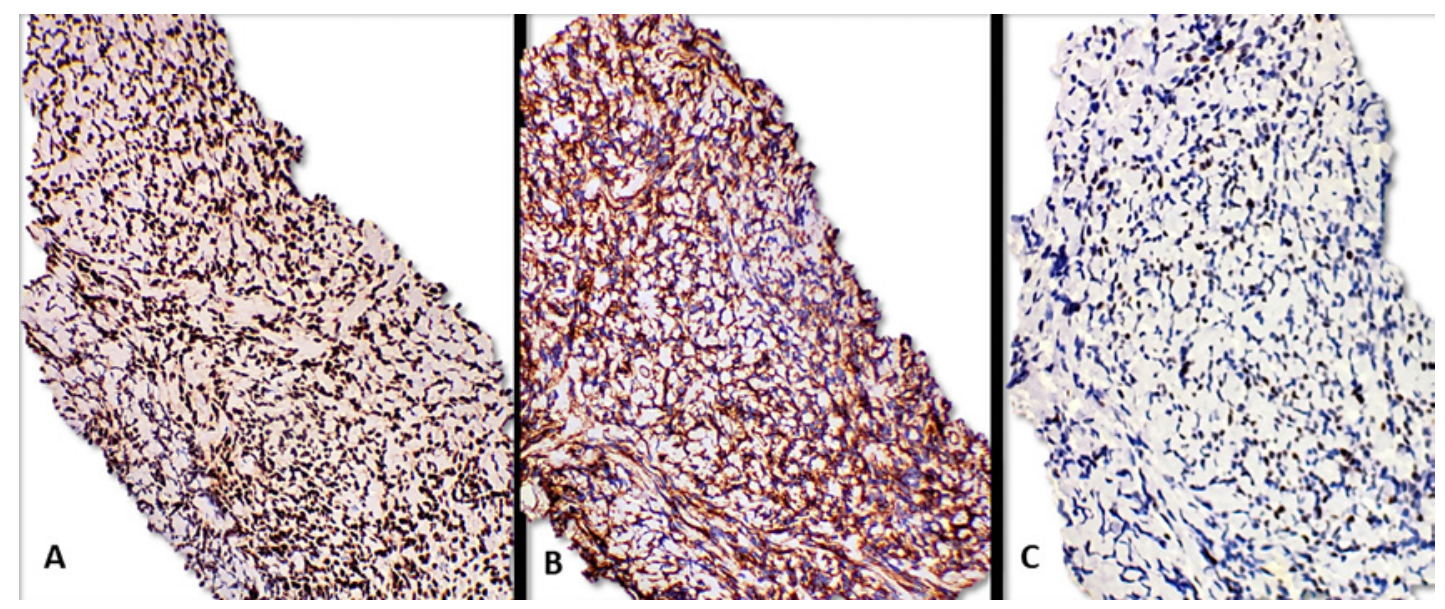

Fig. 3. A Immunostaining for STAT-6 showing strong and diffuse nuclear reactivity. B Immunostaining for CD34 was strongly positive. C TLE-1 showing weak non-specific staining.

\section{Discussion/Conclusion}

The common differential diagnoses of NICTH include SFT, hemangiopericytoma, metastatic hepatocellular carcinoma causing reactive hypoglycemia or hypoglycemia from an ectopic secretion of IGF-II or rarely IGF-I hormone [7-9]. Many other tumors have also been reported to cause NICTH in the literature as listed in Table 1. NICTH associated with SFT is called Doege-Potter syndrome. Han et al. [5] reported that the most common cause of DoegePotter syndrome is SFTP involving the right hemothorax. Of the 71 cases of Doege-Potter syndrome with pathological reports, $43(60.6 \%)$ were malignant. He also noted that extrathoracic SFTs (meningeal, pelvis, bladder, retroperitoneum, etc.) were more likely to be malignant and were frequently associated with hypoglycemia.

Of note, the most common paraneoplastic symptom with SFT is the osteoarthropathy. The prevalence of hypoglycemia is seen in less than $5 \%$ of patients with SFT. It is often secondary to ectopic overexpression of unprocessed precursor "big" IGF-II molecule, which binds to IGF-I receptor (IGF-1R), resulting in the promotion of mitogenesis, tumor growth, and malignant transformation of tumor cells [10]. Although commercial tests to measure serum levels of "big" IGF-II are not available, a sensitive marker suggestive of excessive IGF-II hormone level is the IGF-II/IGF-I ratio. A value of more than 10 suggests excess production of IGF-II and is likely the cause of NICTH $[1,5,10]$.

Histologically, most of the SFTs exhibit a well-circumscribed, uniform, patternless growth of ovoid to spindle cells with varying cellularity, stromal hyalinization, and branching or "staghorn"-shaped thin-walled vessels of varying size and number. Usually, mitotic activity is low ( $<3 / 10$ high-power fields, HPF), cytologic atypia is minimal, and necrosis is infrequent. In contrast, malignant SFTs are usually characterized by the presence of infiltrative margins, nuclear pleomorphism, hypercellularity, mitotic index of $>4 / 10 \mathrm{HPF}$, and necrosis.

A large proportion of lesions are CD34, BCL-2, and CD99 positive, but these markers are non-specific and can be encountered in many soft tissue neoplasms and are, thus, not helpful. Some cases may also be positive with smooth muscle actin, epithelial membrane antigen, S100 protein, keratin, or desmin. A more specific immunohistochemical marker is STAT6, which is positive in $>95 \%$ of cases. 


\section{Case Reports in Oncology}

Table 1. Tumors associated with NICTH

\begin{tabular}{l|l}
\hline Case Rep Oncol 2021;14:470-476 \\
\hline DOI: 10.1159/000512823 & $\begin{array}{l}\text { @ 2 2021 The Author(s). Published by S. Karger AG, Basel } \\
\text { www.karger.com/cro }\end{array}$ \\
\hline
\end{tabular}

\begin{tabular}{lll}
\hline Mesenchymal tumors & Carcinoma & Others \\
\hline Fibrosarcoma & Adrenal cortex & Carcinoid \\
Fibroma & Liver & Hepatoma \\
SFTP & Lung & Lymphoma \\
Hemangiopericytoma & Ovary & Pheochromocytoma \\
Liposarcoma & Colon & Wilms' tumor \\
Leiomyosarcoma & Esophagus & \\
Neurofibroma & Stomach & \\
Mixed lineage carcinosarcoma & Prostate & \\
Mesothelioma & Cervix & \\
& Breast & \\
& & \\
\hline
\end{tabular}

SFTs harbor the characteristic NAB2-STAT6 gene fusion, which results from the intrachromosomal inversion inv(12)(q13q13), leading to the overexpression of STAT6, forming the basis of tumor immunohistochemistry that is currently being used diagnostically. Robinson and colleagues [11] in 2013 identified a recurrent fusion transcript as the main molecular driver on RT-PCR in all 51 patients with SFT (100\%) irrespective of its site of origin or clinical behavior. They noted that NAB2-STAT6 fusion transcripts resulted in the conversion of NAB2 from an early growth response (EGR) repressor to activator driving the differentiation and growth of SFT [11]. Additional fusion variants of NAB2-STAT6 have also been identified; however, no prognostic relevance could be associated with any particular fusion variant in studies so far [12]. In the absence of advanced genome-sequencing techniques, positive immunostaining with STAT6 is often accepted as a marker of nuclear fusion.

Local recurrences have been documented in about $10 \%$ of cases, and the rate of metastasis is said to be around 5-10\% [5]. A mitotic rate of $>4$ mitoses/HPF is the most reliable indicator of aggressive behavior [13]. Our patient's SFT exhibited a well-circumscribed proliferation of spindle cells with a mild-moderate degree of nuclear atypia. There was some central and patchy hyaline degeneration, but no hemorrhage or necrosis was identified on a gross exam or microscopically. Immunohistochemical test with STAT-6 was diffusely positive, supporting the diagnosis of the SFT. Given the features of the tumor on biopsy showing up to 10 mitotic figures/10 HPF focally, in an elderly lady with a large mass $(>15 \mathrm{~cm})$, she was deemed at an intermediate risk for subsequent relapse/metastasis, and a long-term followup was recommended.

The definitive management of Doege-Potter syndrome involves treating the underlying tumor, including complete resection of the tumor mass, palliative debulking, chemotherapy, radiofrequency ablation, cryoablation, or chemoembolization. Until definitive therapy, treatment of hypoglycemia includes increased caloric supplementation, dextrose infusion, glucocorticoids, glucagon, or recombinant human growth hormone (rhGH). Glucocorticoids act by increasing serum big IGF-II clearance and impairing endogenous insulin activity [8]. Few case reports have documented success with oral prednisone 40-60 mg once a day; however, with large tumors and widespread disease, oral dexamethasone was better than prednisone in diminishing the degree of hypoglycemia. For refractory hypoglycemia, continuous infusion of glucagon may be used but is often unsustainable. rhGH use has been successful in some cases but carries a theoretical risk of tumor progression due to an increase in IGF-1 levels [14].

As mentioned above, the current treatment of choice, curative of NICTH, is complete total resection of the tumor. In certain instances, through a reduction in tumor burden, subtotal or partial resection has also resulted in the resolution of Doege-Potter syndrome [15]. Anecdotal reports have demonstrated tumor dormancy and resolution of hypoglycemia with intra-

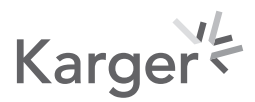


arterial chemotherapy with platinum drugs, anthracyclines [16], and systemic use of tyrosine kinase inhibitors like sorafenib [14]. SFTs are often chemoresistant, and the use of chemotherapy is controversial, reserved mainly for metastatic disease and unresectable masses. Embolization therapy and radiation have not been shown to be effective against SFT. With the discovery of NAB2-STAT6 gene fusion as the hallmark molecular driver of SFT, strategies targeting the inhibition of NAB2-STAT6 downstream pathways could be considered for the treatment of this rare set of neoplasm. Presence of hypoglycemia, large mass, presence of area of necrosis and hemorrhage with high number of mitotic figures and signs of cellular atypia on biopsy have all been noted to be markers of poor prognosis and increase the probability of recurrence in future. Patients with such features often need a long-term follow-up to monitor for disease recurrence.

\section{Statement of Ethics}

The subject of this case report gave her informed consent to publish the case, including the publication of images. No institutional review board or other approvals were required.

\section{Conflict of Interest Statement}

The authors have no conflicts of interest to declare.

\section{Funding Sources}

No funding.

\section{Author Contributions}

Turab Mohammed: managed the patient in hospital, collected data, curated and devised the manuscript. Gonca Ozcan: collected data, wrote part of the manuscript. Ayesha S. Siddique: prepared slides, contributed to the pathological diagnosis view point in the diagnosis part of the manuscript. Dennis E. Slater: as the oncologist on team, helped diagnose and manage the patient. Ronald N. Araneta III: proofread and supervised the findings of the work. Aziz Khan: proofread and finalized the findings of the work. All authors discussed the results and contributed to the final manuscript.

\section{References}

1 Fukuda I, Hizuka N, Ishikawa Y, Yasumoto K, Murakami Y, Sata A, et al. Clinical features of insulin-like growth factor-II producing non-islet-cell tumor hypoglycemia. Growth Horm IGF Res. 2006 Aug;16(4):211-6.

2 Doege KW. FIBRO-SARCOMA OF THE MEDIASTINUM. Ann Surg. 1930 Nov;92(5):955-60.

3 Potter RP. Intrathoracic Tumors. Radiology. 1930 Jan 1;14(1):60-1.

4 Ershadi R, Rahim M, Abbasi M, Erfanian R. Giant solitary fibrous tumor of the pleura. J Surg Case Rep. 2018 Nov;2018(11):rjy270.

5 Han G, Zhang Z, Shen X, Wang K, Zhao Y, He J, et al. Doege-Potter syndrome: A review of the literature including a new case report. Medicine (Baltimore). 2017 Jul;96(27):e7417.

6 NAB2-STAT6 fusions are a hallmark of solitary fibrous tumors. Cancer Discov. 2013 Mar;3(3):0F18.

7 Gorden P, Hendricks CM, Kahn CR, Megyesi K, Roth J. Hypoglycemia Associated with Non-Islet-Cell Tumor and Insulin-like Growth Factors. N Engl J Med. 1981 Dec 10;305(24):1452-5.

\section{Karger's}


8 Bodnar TW, Acevedo MJ, Pietropaolo M. Management of Non-Islet-Cell Tumor Hypoglycemia: A Clinical Review. J Clin Endocrinol Metab. 2014 Mar;99(3):713-22.

9 Nauck MA, Reinecke M, Perren A, Frystyk J, Berishvili G, Zwimpfer C, et al. Hypoglycemia due to paraneoplastic secretion of insulin-like growth factor-I in a patient with metastasizing large-cell carcinoma of the lung. J Clin Endocrinol Metab. 2007 May;92(5):1600-5.

10 de Groot JW, Rikhof B, van Doorn J, Bilo HJ, Alleman MA, Honkoop AH, et al. Non-islet cell tumour-induced hypoglycaemia: a review of the literature including two new cases. Endocr Relat Cancer. 2007 Dec;14(4): 979-93.

11 Robinson DR, Wu YM, Kalyana-Sundaram S, Cao X, Lonigro RJ, Sung YS, et al. Identification of Recurrent NAB2STAT6 Gene Fusions in Solitary Fibrous Tumor by Integrative Sequencing. Nat Genet. 2013 Feb;45(2):180-5.

12 Tai HC, Chuang IC, Chen TC, Li CF, Huang SC, Kao YC, et al. NAB2-STAT6 fusion types account for clinicopathological variations in solitary fibrous tumors. Mod Pathol. 2015 0ct;28(10):1324-35.

13 Travis WD, Brambilla E, Nicholson AG, Yatabe Y, Austin JHM, Beasley MB, et al. The 2015 World Health Organization Classification of Lung Tumors: Impact of Genetic, Clinical and Radiologic Advances Since the 2004 Classification. J Thorac Oncol. 2015 Sep 1;10(9):1243-60.

14 Bourcigaux N, Arnault-Ouary G, Christol R, Périn L, Charbonnel B, Le Bouc Y. Treatment of hypoglycemia using combined glucocorticoid and recombinant human growth hormone in a patient with a metastatic non-islet cell tumor hypoglycemia. Clin Ther. 2005 Feb;27(2):246-51.

15 Yang CY, Chou CW, Hao LJ. Malignant solitary fibrous tumor with hypoglycemia (Doege-Potter syndrome). J Postgrad Med. 2013 Mar;59(1):64-6.

16 Holt RI, Simpson HL, Sönksen PH. The role of the growth hormone-insulin-like growth factor axis in glucose homeostasis. Diabet Med. 2003 Jan;20(1):3-15. 